\title{
Enhanced Large-Area TEM Analysis on Mitochondrial Alteration in Brain Neuronal Cell of Parkinson's Disease Model Mouse Using Montage Function Installed in JEM 1400-Plus System
}

\author{
Hyo-Jeong Kim ${ }^{1,2, \ddagger}$, A Reum Je ${ }^{1,2, \ddagger}$, Mi Jeong Kim ${ }^{1}$, Jae-Kyung Hyun ${ }^{1}$, Hyung-Gun Kim², \\ Yang Hoon Huh ${ }^{1, *}$, Hee-Seok Kweon ${ }^{1, *}$ \\ 1. Division of Electron Microscopic Research, Korea Basic Science Institute, 169-148 \\ Gwahangno, Yuseong-gu, Daejeon, Korea \\ 2. Department of Pharmacology, College of Medicine, Dankook University, 119 Dandaero, \\ Cheonan-si, Chungnam, Korea
}

Despite the important role of transmission electron microscope (TEM) in providing tremendous ultrastructural information of cells, organelles, and molecules [1], one of the disadvantages of TEM is the difficulty associated with observing large-area with high resolution at low-magnification [2 and 3]. Although several analytical techniques and equipments including ultramicrotomy-combined serial block face scanning EM have been developed recently to overcome such problem, those are mostly concentrated in SEM applications [1, 2, 4, 5, and 6]. In this research, to acquire large-area TEM images with highresolution, we used the montage function in TEM Center, a software suite that controls JEM 1400-Plus TEM as described in Figure 1. Then, we compared the resolution of low mag. reference image $(4,000 \mathrm{X})$ acquired using normal imaging mode with that of $3 \mathrm{X} 3$ montage image $(12,000 \mathrm{X})$. The spatial resolution of montage image was improved without any image distortion during montage process (Figure 2). Next, using the montage function, we focused on the morphological alterations of the mitochondria in substantia nigra of hLRRK2 transgenic (G2019S) Parkinson's disease model mice. Normal mitochondria in cell body and axon of wild-type mice mostly contained well-conserved ultrastructure. They maintained dense matrix, compacted with thin and uniform cristae, and surrounded by clear inner and outer membrane. However, majority of mitochondria in cell body and axon of the substantia nigra of hLRRK2 transgenic mice were swollen, different from the structure found in wildtype mice. Also, the mitochondria have deteriorated structure, with the cristae severely disrupted through the entire mitochondrial lumen, and the inner and outer mitochondrial membranes were partially disappeared. This result clearly shows the complex structural alteration of mitochondria in brain neuronal cells of Parkinson's disease model mice, and demonstrates the contribution of montage-based large-area TEM image analysis system for the acquisition of high throughput statistical information for an accurate interpretation of various morpho-functional alterations of cellular organelles [7].

\section{References:}

[1] K Miranda et al., Mol. Reprod. Dev. (2015), doi: 10.1002/mrd. 22455.

[2] E Oho et al., Journal of Electron Microscopy 49 (2000), p.135-141.

[3] Y Ikeda et al., Microsc. Microanal. 19 (2013), p.1330-1331.

[4] C Blumer et al., Medical Image Analysis 19 (2015), p.87-97.

[5] H Horstmann et al., Plos ONE 7 (2012), e35172.

[6] LCS Medeiros et al., Plos ONE 7 (2012), e33445.

[7] This research was supported by Korea Basic Science Institute (\#E35700, T35444) and the Bio \& Medical Technology Developmental Program of the NRF funded by the Ministry of Science, ICT \& 390 Future Planning (\#2013M3A9A9050076). 


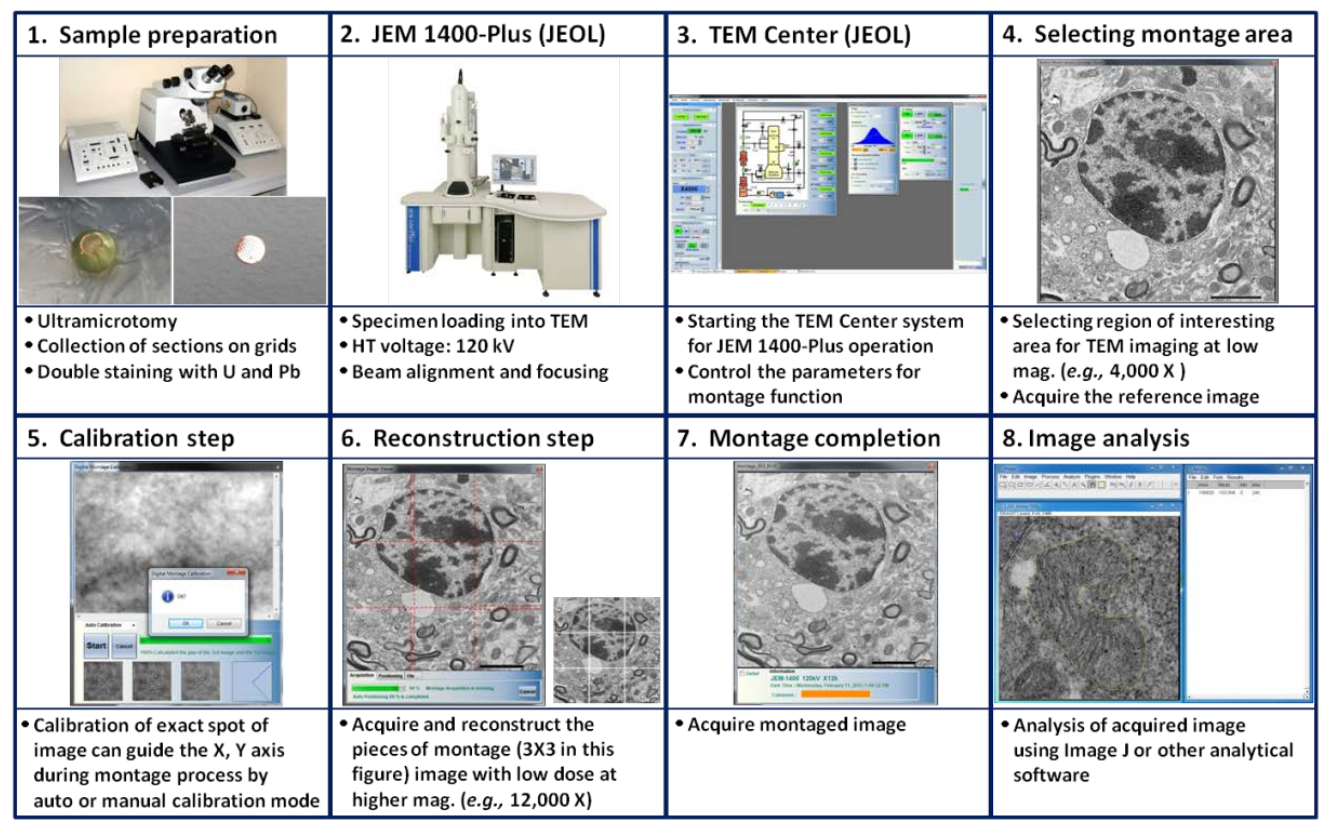

Figure 1. Outline of the experimental procedure for the enhanced large-area EM image acquisition using montage function in TEM Center of JEM 1400-Plus TEM. Simplified flowchart explaining the main experimental steps and conditions for the montage process is described.
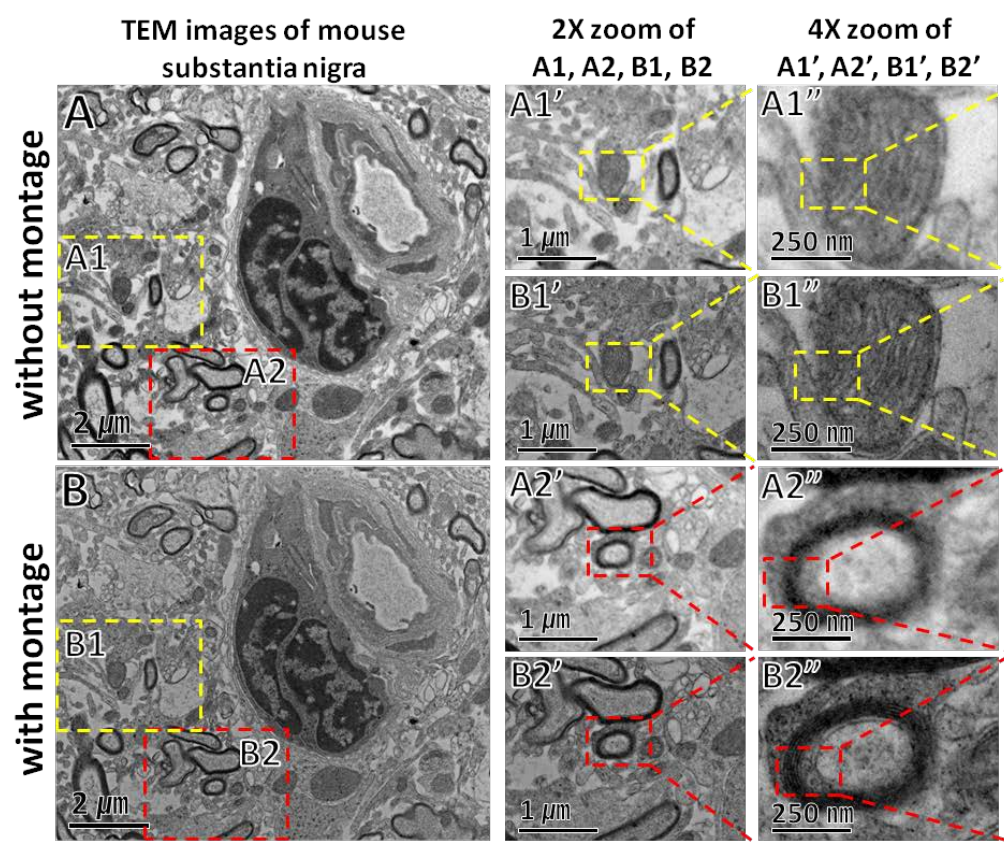

\section{$4 X$ zoom of}
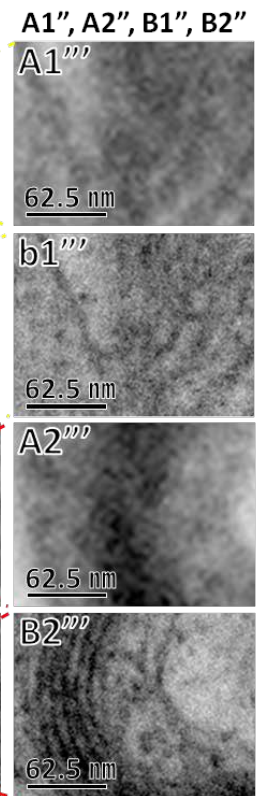

Figure 2. Improvement of the spatial resolution after montage process. The images of mouse substantia nigra without (A) or with (B) montage from same large-area are shown, respectively. A1'-B2': 2X zoomed image of A1-B2. A1"-B2”: 4X zoomed image of A1'-B2'. A1"'-B2"': 4X zoomed image of A1"-B2". 